\title{
EXPERIMENTS ON THE RANDOM FIELD ISING MODEL
}

\author{
D.P. BELANGER \\ Department of Physics, University of California \\ Santa Cruz, CA 95064, USA
}

\begin{abstract}
New advances in experiments on the random-field Ising model, as realized in dilute antiferromagnets, have brought us much closer to a full characterization of the static and dynamic critical behavior of the unusual phase transition in three dimensions $(d=3)$. The most important experiments that have laid the ground work for our present understanding are reviewed. Comparisons of the data with Monte Carlo simulations of the $d=3$ critical behavior are made. We review the current experimental understanding of the destroyed $d=2$ transition and the experiments exploring the $d=2$ metastability at low $T$. Connections to theories most relevant to the interpretations of all the experiments are discussed.
\end{abstract}

\section{Introduction}

The random-field Ising model 1 (RFIM) has been an important focus of theoretical and experimental studies of the statistical physics of random and frustrated systems. Although there are some similarities, particularly at large random fields, to the physics of spinglasses 1 , also covered in this book, the three dimensional $(d=3)$ ground state of the RFIM in the small random-field limit has the same long-range order as would be observed in the absence of random fields. Hence, the two models differ fundamentally. Nevertheless, the $d=3$ RFIM transition is profoundly altered by the random field. For $d=2$ the random field destroys the transition which takes place in the absence of the random field. Not only does the RFIM have significance in the formation of long-range order in real materials, where defects causing random fields are often present, it also challenges the methods and ideas of theorists and experimentalists that have been developed in past studies of phase transitions in pure, translationally invariant materials. There are a number of relevant reviews that have been written covering the formidable problems encountered in the experimental study of RFIM systems 3 . This one represents a comprehensive overview of the experimental situation in the most studied systems, the dilute anisotropic antiferromagnets, emphasizing the most current experimental results. The theories and computer simulations most relevant to interpretations of the behavior observed in dilute antiferromagnets will be included. A few systems that are not antiferromagnets will be mentioned in section 11. A comprehensive review of the theory of the RFIM by Nattermann also appears in this book.

For $d=3$ it has been rigorously 0 shown that a transition must take place for small random fields. As we shall see, the RFIM transition is very different from the more usual phase transitions encountered in antiferromagnets. The RFIM can be most simply modeled by spins on a lattice that point along one axis and are subjected to a random ordering field that competes with the long-range collective spin ordering. One simple Hamiltonian representing an Ising ferromagnet with an imposed random field is

$$
\mathcal{H}=-\sum_{<i, j>} J_{i j} S_{i} S_{j}-\sum_{i} h_{i} S_{i}
$$


The random field has the properties $\left[h_{i}\right]_{a v}=0$ and $\left[h_{i}^{2}\right]_{a v}=h_{r}^{2}$ where $[\ldots]_{a v}$ denotes an average over the disorder. Most of the theoretical and simulation efforts, though not all, have focused on such ferromagnetic models. On the other hand, the most studied and best characterized experimental realization of the RFIM, by far, is the dilute, anisotropic antiferromagnet in a uniform field applied along the spin ordering axis, which can be represented by the Hamiltonian

$$
\mathcal{H}=\sum_{<i, j>} J_{i j} \epsilon_{i} \epsilon_{j} S_{i} S_{j}-\sum_{i} H \epsilon_{i} S_{i}
$$

where $\epsilon_{i}=1$ if site $i$ is occupied and 0 if empty, and $H$ is the uniform field. Locally, the sublattice with the most spins tends to align with the applied field in competition with long-range antiferromagnetic order in which one sublattice globally aligns with the field. The applied uniform field and the effective random field generated by it are proportional 6 . The random field is therefore easily controlled or even turned off completely. This provides the opportunity to do scaling studies not easily done in other systems. Importantly, samples can be cooled in zero field before applying the random field (ZFC). Other systems, such as those with structural phase transitions can only be cooled in the random field (FC). Since, as we shall see, hysteresis plays an important role in the understanding of the RFIM transition, the ZFC process is crucial. Of course, by virtue of critical behavior universality, the systems studied need not correspond precisely to the Hamiltonians above but must simply have the appropriate symmetries.

Fishman and Aharony first noted that the dilute antiferromagnet in a uniform field is a RFIM system and Cardy 6 showed that the critical behavior in the limit of small fields belongs to the same universality class as the uniform ferromagnet with random fields. These works opened up a tremendous opportunity to investigate the RFIM experimentally. An understanding of the RFIM phenomena in the dilute antiferromagnet is steadily evolving with experiments performed on very high quality anisotropic crystals. A major aim of this review is to present an overview of the $d=3$ RFIM transition that takes place in dilute antiferromagnetic systems which is consistent with all of the published data (though certainly not all the published interpretations of the data). The $d=3$ phase diagram has proven much richer than anticipated and this review necessarily encompasses high, intermediate and low magnetic concentrations as well as large and small random fields. The most recent experiments by Slanič, et al. 8 at high magnetic concentrations are promising as they appear to afford the opportunity to make real headway in the experimental characterization of the RFIM critical behavior and in making connections to recent theoretical and simulation results. Such work is still in progress, so only preliminary results can be discussed.

Theory and experiments on the RFIM have been closely tied throughout the period of investigation from the Fishman and Aharony ${ }_{\text {work }}$ until the present, though there has not always been agreement. The greatest progress in the experimental investigations has come when a variety of techniques are employed and interpretations consistent with them are made. Often mistakes have been made when only one technique is relied upon for interpretation. A complication of studies using the dilute antiferromagnet is that random magnetic vacancies constitute strong pinning sites for domain walls 6 . Such strong vacancy pinning, while enriching the $d=3$ antiferromagnetic phase diagram, is not present in the theoretically 
well-studied ferromagnetic model. Random-field pinning, present in both antiferromagnets and ferromagnets, seems to be much weaker. The correspondence between antiferromagnets and the ferromagnetic models is best when the magnetic dilution is small, in which case the antiferromagnetic order is stable up to the transition, $T_{c}(H)$. For concentrations near $x=0.5$, there is evidence that the long-range order breaks into static structure consisting of large, intertwined and weakly interacting domains well below $T_{c}(H)$. This has prevented, at these concentrations, a characterization of $M_{s}^{2}$ vs. $T$ and the line shape below $T_{c}(H)$ When the percolation threshold is approached $\left(x \approx x_{p}=0.25\right)$ a de Almeida-Thouless 10 behavior appears for larger $H$ and the system appears to behave similarly to a spin-glass. In this review each of these three concentration regions is discussed.

For the case of $d=2$ dilute antiferromagnets, the random-exchange Ising model (REIM) transition is expected to be destroyed 11 as soon as $H$, which generates the random field, is applied, and this has been observed 2 , 13 . The temperature regime well below the rounded transition, however, is still being investigated theoretically 14 and experimentally 15 . Both temperature regimes are briefly reviewed.

\section{Sample Considerations}

The most studied dilute $d=3$ antiferromagnet suitable for RFIM studies is $F e_{x} Z n_{1-x} F_{2}$. Its large crystal-field anisotropy persists 16 as the magnetic spins are diluted and it is therefore an excellent Ising system for all ranges of magnetic concentration $x$. Crystals can be grown for all $x$ with extremely small concentration variations $\delta x<10^{-3}$ and with superb structural quality. The magnetic interactions are dominated by the second-nearest- neighbor super-exchange between the body-center and body-corner ions. All other interactions are negligible, except possibly near the percolation threshold concentration, where even tiny frustrating interactions become important 17 18 . Another class of materials representing the anisotropic random-field systems is $\mathrm{Fe}_{x} M g_{1-x} C l_{2}$. This system differs from $F e_{x} Z n_{1-x} F_{2}$ in that it is layered. The layers are ferromagnetic and the interplanar antiferromagnetic coupling is comparable in strength to the intraplane coupling, making this a good $d=3$ Ising system. The smaller exchange in this system allows the large field region of the phase diagram to be explored 19 . For $x<0.55$, a strong second-nearest-neighbor competing exchange in the $\mathrm{Fe}_{x} \mathrm{Mg}_{1-x} \mathrm{Cl}_{2}$ system induces spin-glass behavior 20 and so random-field studies are restricted to higher $x$. There is excellent agreement between the random-field behavior of $\mathrm{Fe}_{x} \mathrm{Mg}_{1-x} \mathrm{Cl}_{2}$ and that of $\mathrm{Fe}_{x} Z n_{1-x} F_{2}$. Some studies have also made use of the highly anisotropic $C o_{x} Z n_{1-x} F_{2}$ system. A number of studies have been made in the less anisotropic system $M n_{x} Z n_{1-x} F_{2}$. The anisotropy in $M n_{x} Z n_{1-x} F_{2}$, which is small for $x=1.0$, decreases further upon dilution. Nevertheless, the $H=0$ REIM critical behavior of $M n_{x} Z n_{1-x} F_{2} 21$ is quite consistent with that of $F e_{x} Z n_{1-x} F_{2} 22$ and all of the RFIM experiments done on $M n_{x} Z n_{1-x} F_{2}$ seem qualitatively consistent with those done in $F e_{x} Z n_{1-x} F_{2}$ and $\mathrm{Fe}_{x} \mathrm{Mg}_{1-x} \mathrm{Cl}_{2}$. The system does allow large applied fields relative to the anisotropy, allowing studies of the spin-flop region 2324. For the $d=2 \mathrm{RFIM}, \mathrm{Rb}_{2} \mathrm{Co}_{x} M g_{1-x} \mathrm{~F}_{4}$ has been studied and appears to be an ideal system 25. It is very anisotropic and consists of layers of magnetic ions with a single dominant intralayer exchange interaction and an interlayer 
interaction which is smaller by several orders of magnitude.

Disagreements among the various interpretations of experimental data have arisen when concentration gradients obscured the true random-field behavior of a sample and were not fully appreciated in the data analyses. Although the gradient effects have been extensively reviewed 26.27, the problem is still relevant to interpretations of recent experiments, as discussed below. Basically, one must realize that a concentration gradient will round a transition and can affect critical behavior measurements drastically. It is best if the gradients are unambiguously determined independently of the critical behavior measuring techniques. The size of the gradient can then be incorporated into the interpretation of the critical behavior data. Disagreements over interpretations of data in RFIM systems are usually resolved once the effect of concentration gradients are properly taken into account.

\section{Scaling Behavior Theory}

Although the scaling behavior of the RFIM has been discussed extensively in previous reviews, we emphasize the salient points again since many experiments are addressing the RFIM critical behavior and, unfortunately, not all of the current experimental interpretations being proposed are consistent with scaling theory. Static critical behavior for temperatures very close to the second-order transition temperature $T_{c}$ can generally be described by power law behaviors which become exact as the reduced temperature $t=T / T_{c}-1 \rightarrow 0$. We briefly list the ones most useful to us. The free energy has the asymptotic behavior $F \sim|t|^{2-\alpha}$, and the specific heat is correspondingly given by

$$
C_{p}=A^{ \pm}|t|^{-\alpha}+B
$$

where we include a constant background term which describes the peak height when $\alpha<0$. For the case where $\alpha \rightarrow 0$ and $A^{+} / A^{-} \rightarrow 1$, we use the symmetric logarithmic form

$$
C_{p}=A \ln |t|+B
$$

instead. Several critical parameters can be obtained from neutron scattering 28 . The correlation length for antiferromagnetic fluctuations has the form

$$
\xi=\kappa^{-1}=\xi_{o}^{ \pm}|t|^{-\nu} .
$$

The staggered susceptibility is

$$
\chi_{s}=\chi_{o}^{ \pm}|t|^{-\gamma} .
$$

For random-field systems we have the disconnected staggered susceptibility

$$
\chi_{s}{ }^{d}=\chi_{o}{ }^{d \pm}|t|^{-\bar{\gamma}} .
$$

The staggered magnetization, the order parameter for antiferromagnets, is given by

$$
M_{s}=M_{o}|t|^{\beta},
$$


which is only nonzero for $t<0$. In these expressions + and - are for $t>0$ and $<0$, respectively. The exponents and the ratios for amplitudes above and below $T_{c}$ are universal parameters common to all systems sharing the same symmetries. The asymptotic critical exponents satisfy scaling relations such as

$$
\gamma+\alpha+2 \beta=2
$$

There are also hyperscaling relations that involve the dimension $d$ such as

$$
\alpha+\nu d=2
$$

that hold for pure and REIM systems but are violated in the RFIM, in which case Eq. 10 is modified 29,30 by the additional violation-of-hyperscaling exponent, $\theta$, with

$$
\alpha+\nu(d-\theta)=2
$$

As the RFIM transition is approached by varying $H$ or $T$, one observes a crossover from the zero-field universality class to the RFIM one. The crossover behavior can be described by a crossover scaling function. For example, the free energy is given by

$$
F=|t|^{2-\alpha} f\left(t h_{r}^{-2 / \phi}\right)
$$

where $\phi$ is the crossover exponent, $\alpha$ is the zero-field exponent and $h_{r}$ is the randomfield strength. A consequence of crossover between different universality class behaviors is that measurements may not yield asymptotic universal parameters unless the data are sufficiently close to $T_{c}$. Rather, one obtains effective exponents. The scaling relations (not the hyperscaling ones) between exponents are still approximately satisfied by the effective exponents 31. Another consequence of the crossover function is a depression of the phase transition temperature given by

$$
T_{c}(H)=T_{N}-A H^{2 / \phi}-b H^{2},
$$

where $b$ represents a small mean-field shift also present in the pure system. The H-T phase boundary curvature is determined by $\phi$. Fishman and Aharony $\mathrm{d}$ showed that for the crossover from pure to random-field $d=3$ behavior, $\phi=\gamma$, with $\gamma=1.25$ obtained from theory and experiment 32 . Although some of the early experiments $23,33,34$ were incorrectly interpreted as showing this, it was also argued 35 that $\phi$ is much larger. The latter result now appears to be universal, with $\phi=1.42+0.03$ obtained for $F e_{x} Z n_{1-x} F_{2} 36, \phi=1.43 \pm 0.03$ for $M n_{x} Z n_{1-x} F_{2} 27$ and $\phi=1.41 \pm 0.05$ for $\mathrm{Fe}_{x} M g_{1-x} \mathrm{Cl}_{2} 37$. Aharony 38 predicted that for a random-exchange to random-field crossover, $\phi$ is several percent larger than $\gamma$. Neutron scattering measurements 22 in $F e_{0.46} Z n_{0.54} F_{2}$ yielding $\gamma=1.31 \pm 0.03$ confirm this. This was similarly verified 21 in $M n_{x} Z n_{1-x} F_{2}$ with the result $\gamma=1.36 \pm 0.08$. The early interpretations 23.33 .34 that $\phi=1.25$ were influenced by the concentration gradients in the samples 26 and the resulting misidentification of $T_{N}$.

The scaling fupction has other consequences for random-field antiferromagnets. Fishman and Aharony $\mathrm{G}$ obtained

$$
k T \chi=A_{1}+A_{2}|t|^{1-\alpha}-A_{3}|t|^{2 \beta}
$$


for the static $H=0$ uniform susceptibility for a system dominated by the pure critical exponents $\beta, \alpha$ and $\gamma$ at $H=0$. The experimental systems, however, are dominated by random-exchange critical behavior at $H=0$ and the correct relationship is therefore 38

$$
k T \chi=A_{1}+A_{2}|t|^{1-\alpha}-A_{3}|t|^{2-\alpha-\phi},
$$

using the REIM exponent $\alpha$ and the REIM to RFIM crossover exponent $\phi$. For $H>0$, Kleemann, et al. 39 showed, by considering leading singularities in derivatives of the free energy for $H \neq 0$, that the field dependence of the amplitude of the peaks in the specific heat, $(\partial M / \partial T)_{H}$ and $(\partial M / \partial H)_{T}$ is governed by the exponents $y=(2 / \phi)(\tilde{\alpha}-\alpha), y=$ $(2 / \phi)(1+\tilde{\alpha}-\alpha-\phi / 2)$, and $y=(2 / \phi)(2+\tilde{\alpha}-\alpha-\phi)$, respectively, where $\tilde{\alpha}$ is the RFIM specific heat exponent. The exponents can be determined from specific heat $\mathrm{\theta}$ and Faraday rotation 39 experiments on $F e_{x} Z n_{1-x} F_{2}$ to be $y \approx 0.1, y=0.60 \pm 0.10$, and $y \approx 0.97$, respectively. For $\mathrm{Fe}_{0.7} \mathrm{Mg}_{0.3} \mathrm{Cl}_{2}, y=0.56 \pm 0.05$ for $(\partial M / \partial H)_{T}$. Since the exponents $\phi=1.42 \pm 0.03$ and $\alpha=-0.09 \pm 0.03$ are known fairly accurately, we can invoke these results as a strong indication from scaling that $\tilde{\alpha} \approx 0$ in good agreement with direct specific heat results discussed below. There is ample evidence that scaling works well in all of these systems, despite recent arguments 41 to the contrary. Hence, at this time, experimental interpretations should be constrained to agree with scaling theory.

\section{The $d=3$ RFIM Transition}

The first evidence that a phase transition occurs in the $d=3$ Ising model came from the critical behavior of the specific heat measured using optical linear birefringence $35(\Delta n)$ techniques, which minimize the effects of concentration gradients since the laser beam is directed perpendicularly to the concentration gradient. The proportionality 4243 between $\frac{d(\Delta n)}{d T}$ and the magnetic contribution $\left(C_{m}\right)$ to the pulsed specific heat $\left(C_{p}\right)$ data has been shown explicitly 84 for $F e_{0.46} Z n_{0.54} F_{2}$ and $F e_{0.93} Z n_{0.07} F_{2}$. In anisotropic systems the Zeeman contribution to the birefringence is negligible at reasonable fields 43 . In addition, Faraday rotation 39 and susceptibility 16 measurements yield the specific heat critical behavior. Recent claims to the contrary 41,45 have no theoretical basis 47 and depend on analyses of experimental data which have been questioned 48 .

The specific heat critical behavior in the intermediate range, $0.4<x<0.8$, has been measured in $F e_{x} Z n_{1-x} F_{2}$ a 49 and $\mathrm{Fe}_{x} \mathrm{Mg}_{1-x} \mathrm{Cl}_{2}$ t0 with enough precision to determine that ZFC data are well described by a symmetric logarithmic divergence over a reasonable range in $t$. At very small $|t|$, rounding is observed and is attributed to the tremendous critical slowing down of the RFIM in dilute antiferromagnets, as will be discussed in the following section. FC always yields behavior that is much more rounded because finitesize metastable clusters 40,50 are frozen in just above $T_{c}(H)$. The region over which the logarithmic ZFC behavior and the dynamic rounding are observed increases with $H$ as expected from crossover scaling (Eq. 12) and dynamic scaling as discussed in the next section.

Figure 1 shows recent $\frac{d(\Delta n)}{d T}$ and $C_{m}$ data for a high magnetic concentration sample 8 $F e_{0.93} Z n_{0.07} F_{2}$. We have subtracted the approximate large phonon contribution to $C_{p}$, leaving only $C_{m}$, so that the correspondence of the two techniques is apparent. We accomplished 

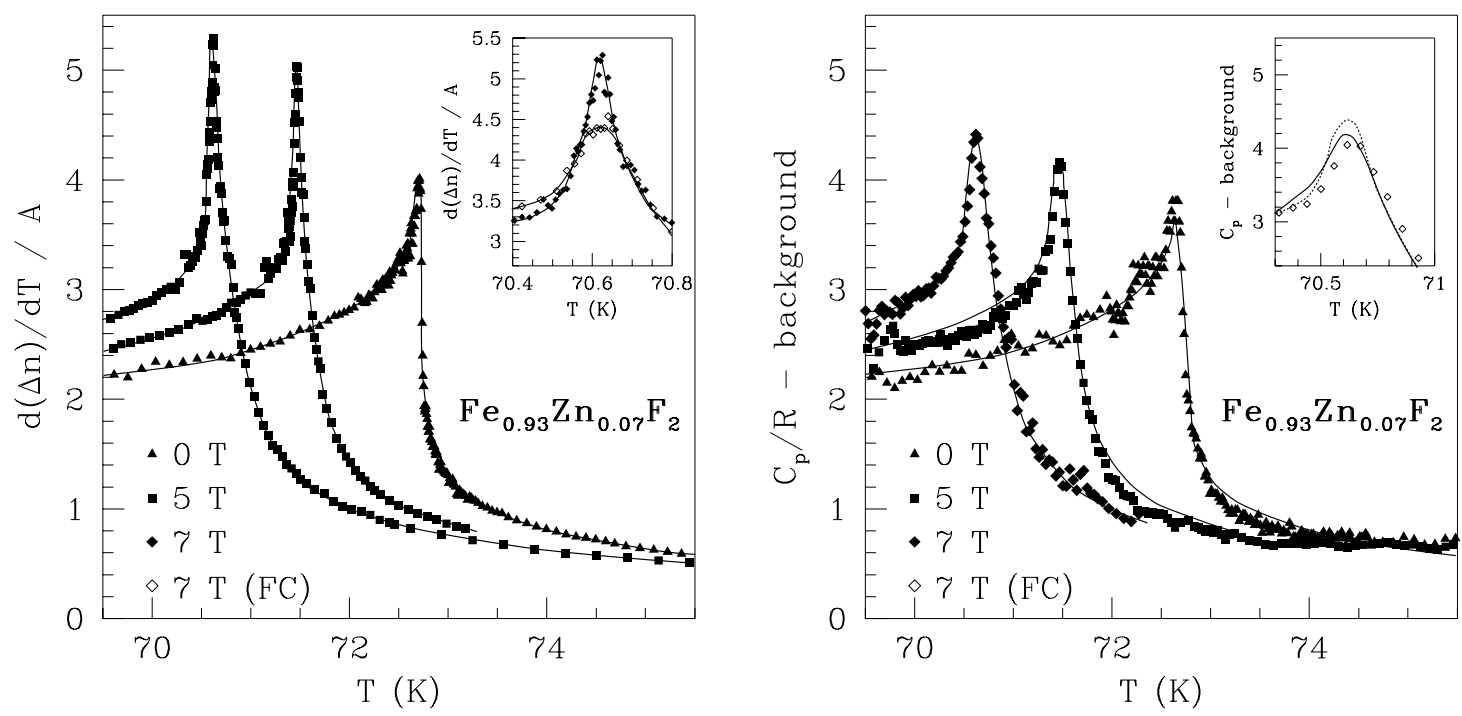

Figure 1: $\frac{d(\Delta n)}{A d T}$ vs. $T$, where $A=9.17 \times 10^{-6} \mathrm{~K}^{-1}$ is the same proportionality constant found for pure $F e F_{2}$, and $C_{m} / R$ vs. $T$ for $F e_{0.93} Z n_{0.07} F_{2}$. The specific heat has the phonon contribution subtracted as discussed in the text. ZFC data are shown in the main figures. The insets show the $H=7 \mathrm{~T} \mathrm{FC}$ data as well as the ZFC data for the $\frac{d(\Delta n)}{d T}$ case. The curves in the left figure are the same as the curves in the figure on the right except that they are rounded by the larger, measured concentration gradient. For the specific heat inset, the ZFC data are not shown, for clarity, but the dotted line is the same as the solid ZFC line in the main figure. Just as in experiments at lower concentrations, the birefringence and pulsed heat techniques yield precisely the same behavior, including FC-ZFC hysteresis very close to $T_{c}(H)$. The critical behavior for $H>0$ is closely approximated by a symmetric, logarithmic divergence.

this by assuming the same proportionality 4 between $C_{m}$ and $\frac{d(\Delta n)}{d T}$ found for both $F e F_{2}$ and $F e_{0.46} Z n_{0.54} F_{2}$. The excess specific heat contribution found for the $H=0$ was then subtracted from all the $C_{p}$ data sets. The solid curves in the $C_{m}$ figure are adapted from those in the $\frac{d(\Delta n)}{d T}$ one by first numerically rounding the $\frac{d(\Delta n)}{d T}$ data by the known gradient, drawing smooth curves through them, and then transferring the curves to the $C_{m}$ figure with no other adjustments. The $C_{m}$ peaks are not as sharp since the entire sample was used, increasing the effects of concentration gradients relative to the $\frac{d(\Delta n)}{d T}$ data which are sensitive only to the gradient along the laser beam. Clearly, the known gradient accounts well for the difference in the $C_{m}$ and $\frac{d(\Delta n)}{d T}$ data. The insets for both sets of data show the $\mathrm{FC}$ behavior at $H=7 \mathrm{~T}$. The FC data are more rounded than the $\mathrm{ZFC}$. The FC $C_{m}$ data are shown in the inset on the right with the $\frac{d(\Delta n)}{d T} \mathrm{FC}$ curve, rounded by the concentration gradient in the same manner as the $\mathrm{ZFC}$ ones, shown as a solid curve. The FC curve corresponds well with the $C_{m}$ data. The dotted curve corresponds to the ZFC and is the same as the solid one in the main figure. For clarity, we do not show the ZFC data in the inset. No other adjustments have been made. Clearly, the hysteresis is much more difficult to discern in the specific heat data 48 , but this is consistent with the larger concentration gradient. The hysteresis can only be observed in samples with extremely small gradients. This is certainly one reason why some experiments on samples with appreciable concentration gradients fail 
to exhibit hysteresis. In all respects we see that the $\frac{d(\Delta n)}{d T}$ and $C_{m}$ data yield the same critical behavior just as was found previously 4 for $F e_{0.46} Z n_{0.54} F_{2}$. As discussed below, $\mathrm{Fe}_{0.93} Z n_{0.07} F_{2}$ yields neutron scattering line shapes that are fundamentally different from those obtained at lower magnetic concentrations in that they do not show hysteresis well below $T_{c}(H)$. Yet, the specific heat appears remarkably similar to that of lower magnetic concentrations. The symmetric, logarithmic $(\alpha=0)$ behavior for ZFC contrasts with the $H=0$ data that are consistent with the expected asymmetric random-exchange 1 cusp.

For all concentrations there is a temperature, $T_{e q}(H)$, below which hysteresis between the FC and ZFC procedures plays a role in the specific heat as well as most other experiments. We emphasize that $C_{m}$ and $\frac{d(\Delta n)}{d T}$ data show precisely the same hysteresis, contradicting recent claims 4 . Using a capacitance technique 52 on $F e_{0.46} Z n_{0.54} F_{2}$ and $F e_{0.72} Z n_{0.28} F_{2}$, the equilibrium boundary $T_{e q}(H)$ has been shown to lie just above $T_{c}(H)$, scaling precisely in the same manner with $\phi \approx 1.42$. The nature of this boundary is still not entirely clear, though it is sharp enough to be measured precisely. It could be related to the extreme critical dynamics discussed in the next section or it could be related to a RFIM spin-glass-like behavior 53,54 between $T_{e q}(H)$ and $T_{c}(H)$. Certainly one must be careful about the data extremely close to $T_{c}(H)$ since the system could be out of equilibrium.

Hysteresis in the specific heat is not well observed in ac techniques 55 used on the less anisotropic system $M n_{x} Z n_{1-x} F_{2}$ at $6.6 \mathrm{~Hz}$. The extremely rapid heating and cooling method (10 K per minute) of measuring specific heat in $\mathrm{Fe}_{x} \mathrm{Mg}_{1-x} \mathrm{Cl}_{2}$ also shows very little hysteresis 34 , although early neutron scattering 56 and measurements in this system clearly exhibit hysteresis. Perhaps the time dependent techniques obscure the difference between FC and ZFC, though this is not yet clear. Recently, it was claimed that in $F e_{0.5} Z n_{0.5} F_{2}$ no hysteresis is observed in the specific heat 11 . Although there is no published description of the procedures used, some conjectures can be made as to why the hysteresis was missed. Perhaps the sample concentration gradient induced rounding of $0.3 \mathrm{~K} 41$ obscures the transition at the low field 6 . The phase boundary might have been exceeded at the high field 48 . 5 . Finally, if the measurements were not sufficiently adiabatic, the hysteresis may be obscured as they appear to be in other time dependent measurements 55,34. The answer is simply unclear at this time and the failure to observe hysteresis could be a combination of effects.

Whereas all the high resolution specific heat measurements done to date indicate a symmetric, logarithmic divergence with no evidence for any accompanying background discontinuity 58 , Monte-Carlo simulations 59.60 indicate a cusp, with a large, negative exponent. This discrepancy between the exponents from simulation and experiment is, as yet, unresolved and is certainly a major challenge to be addressed.

In contrast to the birefringence measurements that first showed evidence 61.35 of a $d=3$ transition, early neutron scattering measurements obtained with the FC process were interpreted as indicating a destroyed transition 62. Upon FC, no Bragg scattering is observed for concentrations $x<0.8$. Instead, a finite-width shape approximated by a squaredLorentzian, as discussed below, appears. We now know that long-range antiferromagnetic order is difficult to establish upon FC at low concentraton, but that a phase transition is nevertheless the basis of the underlying physics. Long-range order does occur for $T<T_{c}(H)$ when the field is applied after ZFC and FC domains have been shown to be metastable 63 . 
Quite different phenomena are observed at high magnetic concentration. Recent scattering measurements 3 using a crystal of $F e_{0.93} Z n_{0.07} F_{2}$ indicate a ZFC transition that is as sharp as allowed by the concentration gradient $\delta x=0.002$. More importantly, there is no evidence of nonequilibrium hysteresis except extremely close to $T_{c}(H)$, as in the specific heat $\mathrm{b}$. What is most remarkable is that the $F e_{0.93} Z n_{0.07} F_{2}$ neutron scattering line shapes show little hysteresis at low $T$. For $x<0.8$, such hysteresis has always been observed and has been a major obstacle to interpreting the critical scattering below $T_{c}(H)$. An important distinction can be made between hysteresis seen in line shapes at lower concentration well below $T_{c}(H)$ which most likely originates in the large number of vacancies, and the rounding near $T_{c}(H)$ that appears even at high magnetic concentration. The latter may well have to do with RFIM critical dynamics as is clearly the case with the specific heat behavior 49 .

The interpretation of the scattering results in RFIM studies is severely hampered by the lack of adequately characterized line shapes provided by theory. As previously reviewed in more detail 364 , mean-field theory yields an elastic scattering cross section of the form

$$
S(q)=\chi(q)+M_{s}^{2} \delta(q)=\frac{A}{q^{2}+\kappa^{2}}+M_{s}^{2} \delta(q),
$$

for a pure system and, with an additional squared-Lorentzian term,

$$
S(q)=\chi(q)+\chi^{d}(q)=\frac{A}{q^{2}+\kappa^{2}}+\frac{B}{\left(q^{2}+\kappa^{2}\right)^{2}}+M_{s}^{2} \delta(q),
$$

for a random-field system 65 . These expressions can be only approximate for $d=2$ or $d=3$ in pure or random systems, as one can see from the required asymptotic behaviors $\chi(0) \sim \kappa^{2-\eta} \sim|t|^{-\gamma}$ and $\chi^{d}(0)-M_{s}^{2} \sim \kappa^{4-\bar{\eta}} \sim|t|^{-\bar{\gamma}}$. The correspondence between the measured line shapes and the mean-field line shapes in pure systems is fairly good for $d=3$ since $\eta \approx 0.04$ is small, though evidence for deviations from mean-field behavior have been observed 32. Although Pelcovits and Aharony 66 predict significant deviations from the Lorentzian line shape for $T<T_{c}(H)$ in the $d=3$ REIM, where $\eta$ is also small, no definitive evidence for this has yet been observed in experiments. For $d=2$, the discrepancy between the line shapes of the pure system and mean-field theory is more evident 67,32 since $\eta=1 / 4$. For random-field systems, $\eta \approx 1 / 2$ is large 3 and the mean-field terms in Eq. 17 are expected to be far from accurate. The observed line shape in the random-field systems is in many cases inconsistent with the Lorentzian in Eq. 16, as was first shown by Yoshizawa, et al.62. However, the story is not as simple as adopting Eq. 17 since this expression is often inconsistent with the data 68, particularly below $T_{c}(H)$. Nevertheless, Eq. 17 is a start.

The first neutron scattering observations of the $d=3$ RFIM phase transition 69, made using $\mathrm{Fe}_{0.6} Z n_{0.4} F_{2}$, showed that above the transition the ZFC line shapes are inconsistent with the single Lorentzian term but can be fit adequately by the sum of Lorentzian and squared-Lorentzian terms as in Eq. 17. Non-Lorentzian line shapes had been observed previously 62 for the metastable domain state well below $T_{c}(H)$ after FC. From the fits to the critical behavior above $T_{e q}(H)$, the estimations $\nu=1.00 \pm 0.03, \gamma=1.75 \pm 0.20$ and $\bar{\gamma}=$ $3.5 \pm 0.3$ are obtained 69 . An attempt at a better characterization, including for $T<T_{c}(H)$, was made 68 with the very homogeneous crystal $F e_{0.46} Z n_{0.54} F_{2}$. Several scans are shown 

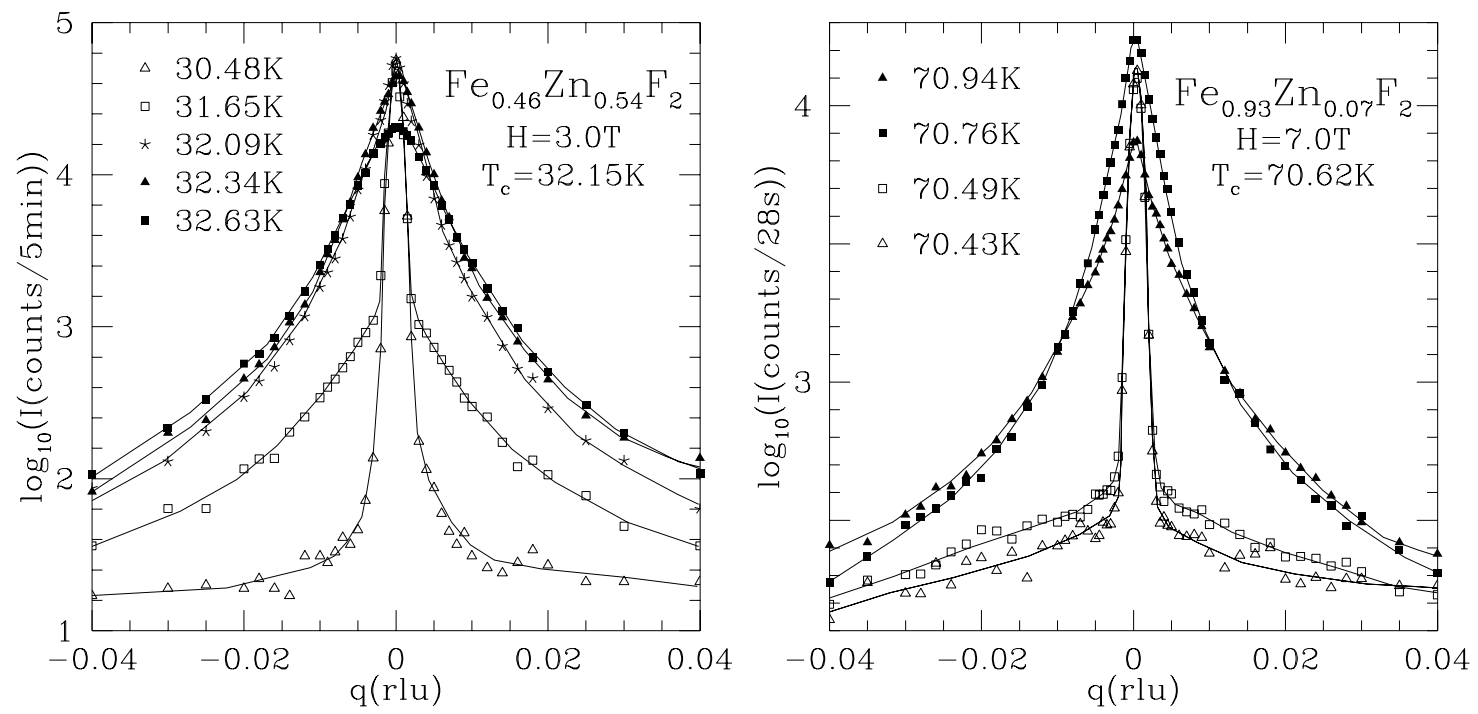

Figure 2: Neutron scattering intensity, $I(q)$ vs. $q$ for $F e_{0.46} Z n_{0.54} F_{2}$ and $F e_{0.93} Z n_{0.07} F_{2}$ after ZFC. Above $T_{c}(H)$, determined from the peak in the critical scattering, both samples exhibit Lorentzian plus squaredLorentzian line shapes. Below $T_{c}(H)$, the $F e_{0.46} Z n_{0.54} F_{2}$ crystal shows a resolution limited lineshape which conforms to neither a Lorentzian nor a squared-Lorentzian line shape in addition to the Bragg scattering peak. The Bragg scattering decreases dramatically at this concentration as $T_{c}(H)$ is approached and shows a large hysteresis upon temperature cycling below $T_{c}(H)$. This behavior is consistent with the system breaking into large, intertwined, weakly interacting domains, a result of the very large number of vacancies at this concentration. In contrast, the $F e_{0.93} Z n_{0.07} F_{2}$ crystal exhibits Lorentzian line shapes below $T_{c}(H)$ with no sign of domain formation. No hysteresis for $|q|>0$ is observed well below $T_{c}(H)$, indicating equilibrium behavior. The Bragg intensity remains large extremely close to $T_{c}(H)$, indicating that $\beta$ is very small, in agreement with simulations.

in Fig. 2. Although the ZFC scattering above $T_{c}(H)$ is indeed fairly well fit by Eq. 17, the scattering line shapes below $T_{c}(H)$ are certainly not. Below $T_{c}(H)$ the measured scattering profiles are much too narrow, being essentially resolution limited for all $T<T_{c}(H)$ instead of having a width that varies as $\kappa(T)$. Furthermore, the intensity of the Bragg component is surprisingly small near $T_{c}(H)$ and qualitatively it appears as if the Bragg peak transforms into the non-Lorentzian, resolution-limited scattering contribution as $T_{c}(H)$ is approached from below. Finally, a peak in the $q=0$ scattering intensity is observed 68 at $T_{c}(H)$ upon ZFC and the height of the peak grows approximately logarithmically with time, a result of the extremely slow dynamics. Such a peak is difficult to observe normally but is evident in this case because the Bragg scattering, which usually swamps the critical fluctuation peak, is abnormally small just below $T_{c}(H)$.

Although it is clear that the scattering is peculiar and interesting, extinction effects 70 give pause to direct interpretations of the Bragg scattering intensities in the $F e_{0.46} Z n_{0.54} F_{2}$ experiments. High quality crystals scatter neutrons that are precisely aligned for the Bragg scattering condition in the first ten microns or so of material. As the scattering cross section diminishes upon approaching $T_{c}(H)$, the scattering simply occurs over a larger volume. Hence, the scattered intensity is saturated and does not exhibit the power law 
behavior in Eq. 8. This difficult problem has been overcome by examining 71 an epitaxial $F e_{0.52} Z n_{0.48} F_{2}$ film of thickness $3.4 \mu \mathrm{m}$, grown on a $Z n F_{2}$ substrate. The film is thin enough to avoid extinction effects but thick enough $\left(\approx 10^{4}\right.$ lattice spacings $)$ for $d=3$ critical behavior.

The neutron scattering results for the $H=0$ Bragg intensity 71 of the film are consistent with REIM behavior. Hence, the film is high enough 72 in quality to reliably reflect the $d=3$ critical behavior. The scattering intensity for $q>0$, coming solely from a Lorentzian contribution, is too weak to be observed in the film. For $H>0$ the scattering results are highly unusual. The ZFC Bragg intensity vs. $T$ has the opposite curvature to that observed for $H=0$, so the Bragg scattering intensity is very small quite far below $T_{c}(H)$. The loss of the ZFC Bragg intensity is irreversible below $T_{c}(H)$. This behavior has been interpreted as the system breaking into two intertwined domains with equal numbers of spins in a similar pattern to that observed in FC simulations 3 at low $T$. The formation of domains is observed to be irreversible below $T_{c}(H)$, a result that is consistent with the irreversibility observed in magnetization and optical studie 4,43 . Inside the domains the spins are well ordered. The domain walls at this concentration $(x \approx 0.5)$ are able to pass predominantly through the numerous vacancies, costing the system very little energy. It is clear that the Imry-Ma domain wall energy arguments 1 fail here since the energy needed to create such a domain wall is insignificant compared to the Zeemann energy decrease. Furthermore, the domains are only weakly interacting and each contributes to the phase transition at $T_{c}(H)$. Since the domains form well below $T_{c}(H)$, neutron scattering measurements are unable at this concentration to determine the critical behavior of the order parameter. Another piece of evidence indicating that the hysteresis for $T_{c}(H)$ comes from domain formation is found in the experimental results of x-ray scattering studies 75 at the surface of $M n_{0.75} Z n_{0.25} F_{2}$. In the presence of surface defects, no hysteresis is observed, most likely a result of the defects preventing the formation of the two intertwining domains. When an identical sample was polished, removing the majority of defects, the hysteresis reappeared.

In both the film $\left(F e_{0.52} Z n_{0.48} F_{2}\right)$ and bulk $\left(F e_{0.46} Z n_{0.54} F_{2}\right)$ studies, we find a large resolution-limited scattering line shape below the transition that is not well fit by either a Lorentzian or squared-Lorentzian term. It is most likely that this non-Lorentzian scattering profile is a signature of domain structure that forms below $T_{c}(H)$ even upon heating after ZFC. With this structure present it is very difficult to determine the critical behavior of the RFIM below the transition. Local probes like NMR 6 , Mössbauer or $\mu$ SR in principle could yield the order parameter critical behavior, but prove to be complicated because of the spatial variations within the system. This motivated an investigation at a much higher concentration, where the vacancy concentration is small enough that domain walls cannot easily avoid a large energy cost of formation. Since hysteresis at low temperatures is seen in the work 73 on $M n_{0.75} Z n_{0.25} F_{2}$, it is clear that one must go to even higher concentrations. Preliminary measurements $\mathrm{G}$ using the $F e_{0.93} Z n_{0.07} F_{2}$ crystal seem to confirm the idea; the hysteresis in the scattering profile at low temperatures is eliminated.

The abrupt change in line shape of $F e_{0.93} Z n_{0.07} F_{2}$ at $T_{c}(H)$ is striking. Figure 2 shows scans taken just above and just below $T_{c}(H)$ at $H=7 \mathrm{~T}$. Just $0.13 \mathrm{~K}$ below $T_{c}(H)$ the line shape is incompatible with any significant squared-Lorentzian term. A Lorentzian term fits 

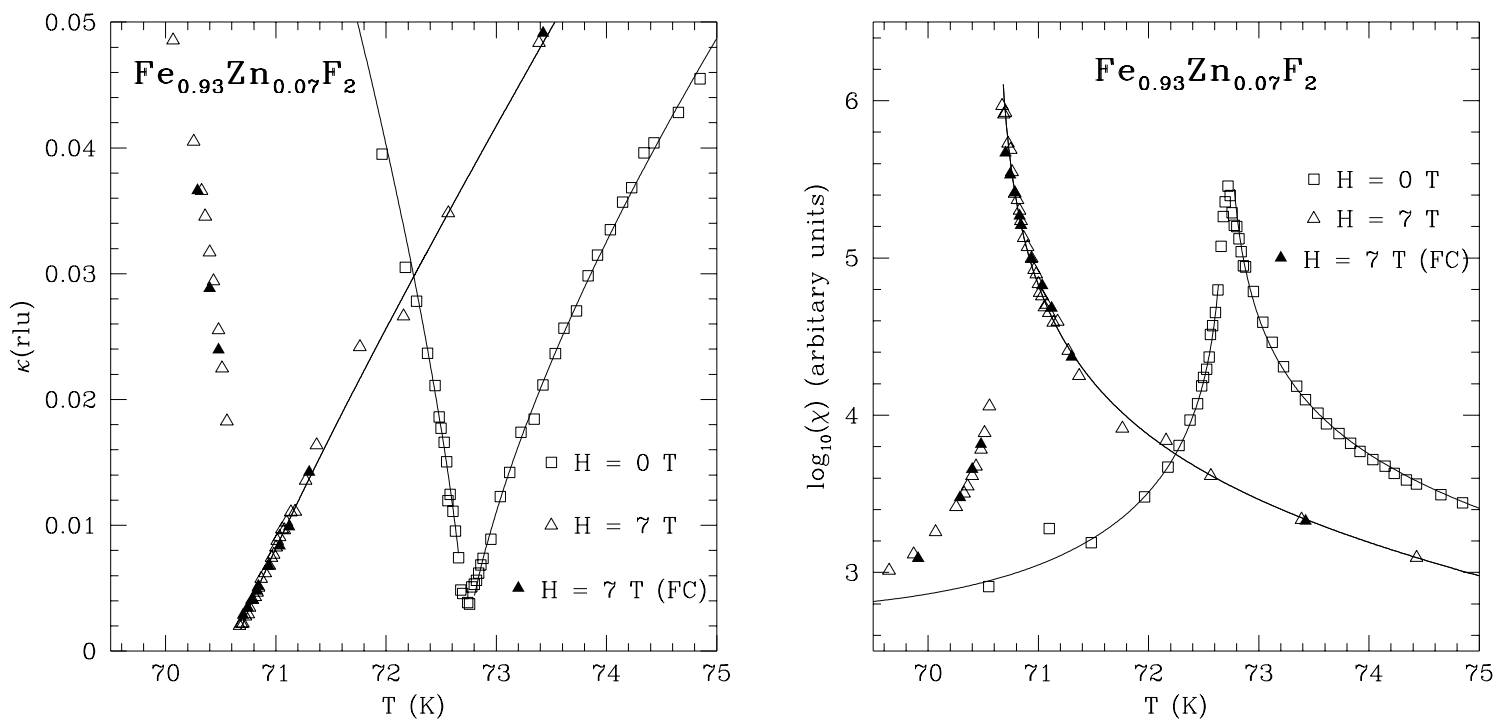

Figure 3: $\kappa$ vs. $T$ and $\chi$ vs. $T$ for $F e_{0.93} Z n_{0.07} F_{2}$ for $H=0$ and $7 \mathrm{~T}$, obtained from preliminary analysis of the neutron scattering line profiles for $|q|>0$. For $H=7 \mathrm{~T}$ and $T>T_{c}(H)$, the $|q|>0$ scattering is fit to a Lorentzian plus squared-Lorentzian lineshape. A Lorentzian was used in all other cases. For $H=7 \mathrm{~T}$, the open triangles are for ZFC and the filled ones are for FC. The lack of hysteresis indicates equilibrium behavior. The solid curves are fits to the data. However, for $H=7 \mathrm{~T}$ and $T<T_{c}(H)$, no power law describes the data well, so no curve is shown for this case.

fairly well. The absence of the non-Lorentzian component is most likely a signature of the stability of the long-range order right up to $T_{c}(H)$. Above $T_{c}(H)$, on the other hand, the line shapes are much more compatible with a fit to a Lorentzian plus squared-Lorentzian as in Eq. 17. The abrupt disappearance of the Bragg peak at $T_{c}(H)$, indicating a very small value for $\beta$, contrasts greatly the behavior observed at lower concentrations in $F e_{x} Z n_{1-x} F_{2}$. A small value of $\beta$ is consistent with theory and simulation results 59.60 .68 . The only previous experimental measurement 79 of $\beta$ is from dilation experiments on the lower concentration sample $F e_{0.46} Z n_{0.54} F_{2}$ which indicates $\beta \leq 1 / 8$. This suggests that the small exponent value holds for lower concentrations even though the neutron scattering Bragg intensity cannot show it. The small value of $\beta$ is perhaps suggestive of a first-order transition. but no latent heat is observed in the specific heat in the experiments or simulations 44.80 .

At the lower magnetic concentrations, severe hysteresis is observed in the line shapes below $T_{c}(H)$. In the case of $F e_{0.93} Z n_{0.07} F_{2}$, however, the line shapes for $q>0$ do not exhibit hysteresis except for the region near $T_{c}(H)$ where critical dynamics dominate. The Bragg intensity does show some hysteresis, being somewhat larger upon FC, but this is an extinction effect 70 reflecting the fact that long-range order on length scales well beyond the instrumental resolution is not established upon FC, most likely a result of RFIM dynamics very close to $T_{c}(H)$. The temperature dependence of the Bragg intensity is essentially the same for the Bragg intensity upon ZFC and FC well below $T_{c}(H)$.

Evidently, if we can extract the critical behavior in the $F e_{0.93} Z n_{0.07} F_{2}$ sample, it should 
represent the equilibrium behavior since it is history independent. Unfortunately, the lack of a theoretical scattering line shape that goes beyond the misleading mean-field theory of Eq. 17 has severely limited the extraction of critical parameters below $T_{c}(H)$. Experimental work in this area is ongoing with progress anticipated, but theoretical work is also much needed in the near future. Above the transition the fits to Eq. 17 seem to work fairly well and one can extract the exponents, albeit with trepidation regarding exact results. The results for $\kappa$ and $\chi$ vs. $T$ are shown in Fig. 3 along with fits represented by the solid curves. Fits were made for all of the $H=0$ data and for $T>T_{c}(H)$ with the $H=7 \mathrm{~T}$ data. No suitable fit to a power law is obtained for $T<T_{c}(H)$ and no curves are shown. Preliminary fits $\mathrm{G}$ for $T>T_{c}(H)$ yield $\nu=0.93 \pm 0.03, \gamma=1.71 \pm 0.06$ and $\bar{\gamma}=3.0 \pm 0.1$ for $10^{-3}<t<10^{-2}$. These values are in reasonable agreement with earlier experimental results 69 at $x=0.6$ mentioned above but are in disagreement with other estimations where the transition appears distinctly rounded 81 from concentration gradients. (Larger values for $\nu$ have been obtained in other studies, but only because $T_{c}(H)$ has been taken to be well below the minimum in $\kappa$ in samples with relatively large gradient induced rounding.) There is reasonably good agreement between the exponents obtained from neutron scattering in $\mathrm{Fe}_{0.93} \mathrm{Zn}_{0.07} F_{2}$ and those obtained from Monte Carlo simulations. For example, Rieger 60 obtains $\nu=1.1 \pm 0.2, \gamma=1.7 \pm 0.2, \bar{\gamma}=3.3 \pm 0.6$, and $\beta=0.00 \pm 0.05$ for a Gaussian distribution of random fields. Thescattering results are also reasonably consistent with recent high temperature expansion 82 results for $\gamma$ and $\bar{\gamma}$.

Keeping in mind the uncertainty concerning the scattering line shape appropriate for analyzing the $\mathrm{Fe}_{0.93} Z n_{0.07} F_{2}$ data, the preliminary scattering exponents above $T_{c}(H)$, $\gamma=1.71$ and and from the specific heat, $\alpha \approx 0$, satisfy the simple scaling relation in Eq. 9 if $\beta$ is small as expected from theory. In stark contrast, a typical result from Monte Carlo simulations is that $\alpha$ is large and negative, for example $\alpha=-0.5 \pm 0.260$. Nevertheless, the specific heat exponent is the most consistent experimental exponent. Note that the measured amplitude ratio $A^{+} / A^{-}$is very close to unity which is consistent with a logarithmic divergence. Also, as demonstrated in section 3 , amplitude scaling relations for dilute antiferromagnets strongly indicate $\alpha \approx 0$.

We can use Eq. 11 and the measured exponent $\nu=0.93$ for $T>T_{c}(H)$ to estimate the violation of hyperscaling exponent $\theta=0.85$. Using the relations $\gamma=\nu(2-\eta)$ and $\bar{\gamma}=\nu(4-\bar{\eta})$ with the values from scattering $\gamma=1.71$ and $\bar{\gamma}=3.0$, we can estimate $\eta=0.16$ and $\bar{\eta}=0.77$. These values are smaller than theoretical estimates, but they are very preliminary and further measurements and analysis will certainly refine them in the near future. The point to be made is that we are finally almost at the stage where serious comparison with theory can be made, though we are greatly hampered by not knowing the correct line shape.

Finally, we should briefly mention a very recent suggestion by Birgeneau, et al. 41.65 that the unusual curvature of the Bragg intensity versus $T$ is actually a rounding of the phase transition at intermediate concentrations - the "trompe l'oeil" phenomenological model, as they have labelled it. It was introduced in an attempt to describe the scattering, magnetization and specific heat behavior of the $d=3$ RFIM phase transition in the lower concentration antiferromagnets $F e_{0.5} Z n_{0.5} F_{2}$ and $M n_{0.75} Z n_{0.25} F_{2}$. The interpretation of 


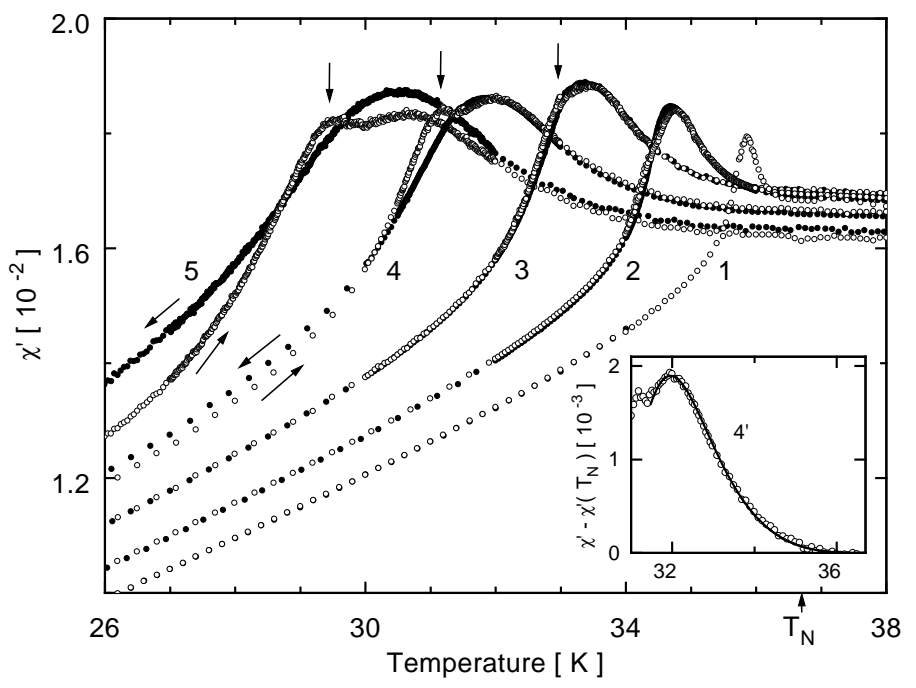

Figure 4: $\chi^{\prime}$ vs. $T$ at $f=1 \mathrm{~Hz}$ for $F e_{0.47} Z n_{0.53} F_{2}$ for applied fields $H=0.8,1.6,2.4,3.2$ and $4 \mathrm{MA} / \mathrm{m}$. Open circles are ZFC data and filled circles are FC. The main contribution to the peak is from Griffiths-like clusters which form above $T_{c}(H)$. The smaller peak, which is resolved only at larger fields and only upon FC, is at the actual phase transition. From the dependence of the small ZFC peak height vs. the frequency, the dynamics can be ascertained. The behavior is consistent with a power law with a very large dynamic exponent or with activated dynamics. The inset shows a fit to the broad peak at $H=3.2 \mathrm{MA} / \mathrm{m} \mathrm{using}$ a phenomenological Griffiths-cluster model.

the data in this model conflicts sharply with the interpretations presented in this review 4 , since it clearly violates scaling for $H>0$, which was developed by Kleemann, et al. 39 and Fishman and Aharony and is described in section 3. The authors take this as evidence that the scaling theory is incorrect. The interpretation requires that the peak in $(\partial M / \partial T)_{H}$ coincide with the peak in $\left(\partial M_{s}^{2} / \partial T\right)_{H}$ and, to accomodate this, the $\left(\partial M_{s}^{2} / \partial T\right)_{H}$ data are adjusted within the thermometry uncertainties. The shifts of the data weaken the motivation for the new model and the argument that scaling fails. The proposed model requires that the uniform magnetization couple strongly to the antiferromagnetic long-range order and this has not yet found theoretical motivation 4 . The model also depends on the specific heat in $F e_{0.5} Z n_{0.5} F_{2}$ showing no hysteresis, but such hysteresis has been observed in pulsed heat experiments using $F e_{0.46} Z n_{0.54} F_{2}$ and $F e_{0.93} Z n_{0.07} F_{2}$ 䀚 crystals with very small concentration gradients.

\section{Critical Dynamics of the $d=3$ RFIM Transition}

The critical dynamics of the $d=3$ RFIM transition are extraordinarily slow. Many of the experiments in $F e_{x} Z n_{1-x} F_{2}$ for $H>0$ that would normally be considered static measurements have shown behavior with approximately logarithmic time dependence. These include neutron critical scattering 68 and capacitance techniques 2 . Spin-echo neutron scattering techniques show 83 that very small fields suffice to freeze the system over the entire 
critical region $|t|<0.1$ in the nanosecond time regime. The most direct measurements of the RFIM critical dynamics are of the peak height of the ac susceptibility. The first susceptibility measurements on a RFIM antiferromagnet were performed on $\mathrm{GdAlO}_{3}: \mathrm{La}$ by Rohrer 84 . Although at the time it was thought that the very rounded transition was evidence of the destruction of the $d=3$ RFIM transition, it is now understood that the rounding is caused by slow dynamics. King, et al. 55 measured the peak height in the ac susceptibility of $F e_{0.46} Z n_{0.54} F_{2}$ as a function of frequency and showed that the behavior is consistent with either a power law behavior

$$
\chi^{\prime}(\omega) \sim|t|^{-\alpha} F\left(\omega|t|^{\nu z}\right),
$$

where $z \nu \approx 14$ has an unusually large value, or with activated dynamics with

$$
\chi^{\prime}(\omega) \sim|t|^{-\alpha} G\left(\ln \omega^{\theta}\right),
$$

where $\theta$ is the violation-of-hyperscaling exponent (Eq. 11), as predicted by Villain 29 and Fisher 30. Later Nash, et al. 86 extended the measurements on the same sample to a very large frequency range of $5 \times 10^{-3} \leq \omega / 2 \pi \leq 10^{5} \mathrm{~Hz}$ and showed that activated dynamics are favored by the data with $\theta=1.05 \pm 0.2$. This is in accord with the violation of hyperscaling relation $(d-\theta) \nu=2-\alpha$ using the measured values of $\nu \approx 1$ and $\alpha \approx 0$, though it has been suggested that corrections to scaling should be considered 87 . However, the picture changed substantially when, recently, Binek, Kuttler and Kleemann 16 demonstrated that in $F e_{0.47} Z n_{0.53} F_{2}$ the peak in the ac susceptibility studied previously is not that of the phase transition itself but rather is due primarily to the dynamics of Griffiths-like spatial fluctuations 88 above $T_{c}(H)$. It was shown that the true critical peak corresponding to the phase transition is but a small peak that is not resolved at low fields and was therefore missed in earlier studies, as shown in Fig. 4. The shape of the peak is consistent with the exponent $\alpha \approx 0$ obtained in other experiments. High resolution measurements for a frequency range $3 \times 10^{-1} \leq \omega \leq 3 \times 10^{3} \mathrm{~Hz}$ again show that the peak is adequately described by either the power law with an unusually large exponent, $z \nu \approx 14$, or with activated dynamics. The critical peak, visible only upon ZFC, is surprisingly weak, indicating that only a small portion of the spins are involved in the phase transition. This is consistent with the very small peak observed in specific heat experiments at this concentration 4 . The larger peak has been related 16 to Griffiths-like instabilities in the temperature range between $T_{c}(H)$ and $T_{N}$, as discussed in the next section. Further refinement of the theory for the ac susceptibility peaks and investigation of other samples, for example $F e_{0.93} Z n_{0.07} F_{2}$, may eventually settle the question of which dynamic model best fits the $d=3$ RFIM in dilute antiferromagnets. The unusual RFIM dynamics have also been observed 89 in $\mathrm{Fe}_{0.7} \mathrm{Mg}_{0.3} \mathrm{Cl}_{2}$ using Faraday rotation techniques, where a symmetric logarithmic peak is seen with rounding. A fit of the peak height to a power law behavior yields $z \nu=8.3$.

\section{The $d=2$ Destroyed RFIM Transition}

In contrast to the $d=3$ case, it is clear from theory 11 that the $d=2$ phase transition is destroyed by the random field. Experimentally this was demonstrated definitively 

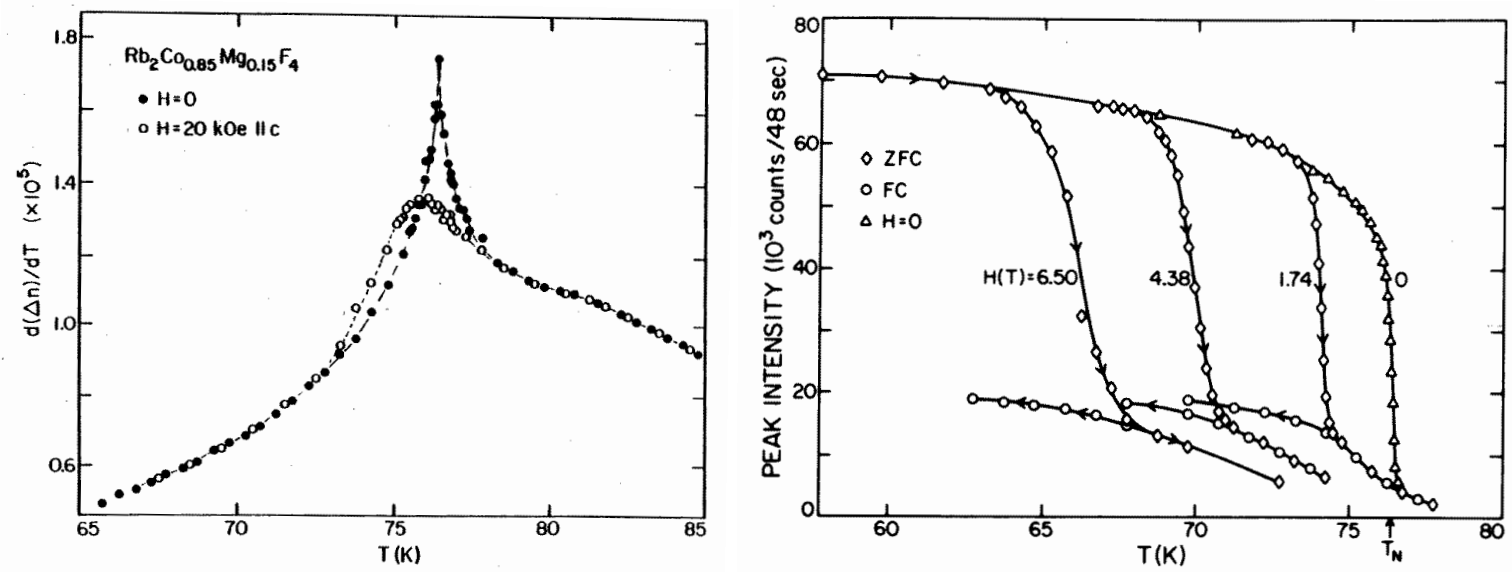

Figure 5: $d(\Delta n) / d T$ vs. $T$ and the $q=0$ peak intensity vs. $T$ for the $d=2$ RFIM system $R b_{2} C o_{0.85} M g_{0.15} F_{4}$. The birefringence data show that the application of the random field destroys the transition. The data show no hysteresis near $T_{c}(H)$, which indicates equilibrium behavior. The neutron scattering peak intensities are obtained after ZFC and FC. At low temperatures the long-range antiferromagnetic order Bragg component is stable. As $T$ is increase, the long-range order becomes unstable and decays, well below the temperature region of the destroyed phase transition. No long-range order is observed upon FC.

in $R b_{2} C o_{x} M g_{1-x} F_{4}$ by the birefringence experiments of Ferreira, et al. 12 and $(\partial M / \partial T)_{H}$ experiments of Ikeda 3 . $\frac{d(\Delta n)}{d T}$ is proportional to the magnetic specific heat 33 and is particularly important for low dimensional systems where the phonon specific heat is considerable. The transition for $H=0$ is well described experimentally by a symmetric logarithmic divergence. However, even relatively small applied fields round the transition, as is evident in Fig. 5. This behavior contrasts greatly with $d=3$ rounding observed upon FC since the $d=2$ crystal is in equilibrium above and below the $H=0$ transition and no hysteresis is observed upon FC and ZFC. As the field increases, the rounding also increases in a way consistent with the random-field scaling function (Eq. 12) with a crossover exponent $\phi=1.75$, which is approximately equal to the zero-field staggered susceptibility exponent 90 as expected.

Whereas the behavior near $T_{N}$ is in excellent accord with theory, the physics of $d=2$ dilute antiferromagnets at low temperatures may not be equivalent to that of the ferromagnet with random fields 91,92. The low $T$ behavior is discussed in the next section.

The neutron scattering line shapes were studied when the sample was FC to temperatures well below the destroyed phase transition 93 where nonequilibrium behavior dominates. The Lorentzian plus squared-Lorentzian line shape of Eq. 17 works quite well as does a Lorentzian to a power of approximately $3 / 2$. Although no comprehensive study has been made of the line shapes near $T_{c}(H)$, preliminary indications are that the squared-Lorentzian scattering term is relatively unimportant in $\mathrm{Rb}_{2} \mathrm{Co}_{0.85} \mathrm{Mg}_{0.15} \mathrm{~F}_{4}$ in this equilibrium region 94 . This conflicts with the mean-field theory that predicts that the squared-Lorentzian should be just as important for $d=2$ and $d=3$ near $T_{c}(H)$ and suggests that the mean-field arguments for the squared-Lorentzian are not particularly relevant. This problem deserves further study. 


\section{Low Temperature Dynamics in $d=3$ and $d=2$}

The dynamics of the RFIM below $T_{c}(H)$ in dilute antiferromagnets have been explored using Squid magnetometry in $F e_{0.46} Z n_{0.54} F_{2}$ by Lederman, et al. 95 After inducing domains using the FC procedure, the relaxation of the metastable domain walls has been measured as a function of time. The excess magnetization from the domain walls scales as the inverse of the domain size 74. The dynamics for a variety of fields and temperatures have been characterized. Below $T_{2}(H)$, which is approximately equal to the equilibrium line $T_{e q}(H)$, and above the another line $T_{1}(H)$, the time dependence of the domain wall size $R(t)$ is consistent with the expression introduced by Villain 96, $R(t) \sim H^{-\nu_{H}} \ln (t / \tau)$, where $\tau$ is a spin-flip time. This indicates that the dynamics are governed by the pinning from the random-field fluctuations. Below $T_{1}(H)$ at lower fields, the random-field pinning seems to be insignificant relative to the pinning from vacancies, which are known to freeze in domain structure even at zero field 49.73 for $d=3$. At very low $T$ for all fields the Ising character of the spins is sufficient to freeze the domain structure. It is not yet clear how this picture might change with variation in the magnetic concentration. The time dependence observed by Lederman, et al. is consistent with recent domain growth near $T_{c}(H)$ observed by Feng, et al. 97 in $\mathrm{Fe}_{0.5} Z n_{0.5} F_{2}$ in a very large 57 field $H=5.5 T$. The line shape width decreases with time near $T_{c}(H)$ but not at low temperatures. The smaller field behavior has not been probed. RFIM dynamics have also been observed using Monte Carlo techniques 98 .

The low $T$ dynamics of the $d=2$ RFIM dilute antiferromagnet $R b_{2} C_{0.85} M_{0.15} F_{4}$ were probed using neutron scattering techniques 99 . No Bragg peak develops upon cooling with $H>0$ since the equilibrium phase transition is destroyed 12 . Instead, a non-Lorentzian-like scattering line shape develops 62 well below the rounded transition. On the other hand, if the system is ZFC, long-range order is observed to be stable at low $T$ for $H>0$. Upon heating, a temperature region is reached where the Bragg scattering peak decays. This region, shown in Fig. 5, is well below the destroyed phase transition as seen by comparing with the birefringence data 12 , also in Fig. 5. The time dependence of the decay of the Bragg intensity at the steepest slope in the Bragg intensity, $T_{F}$, versus $T$ is observed to be approximately logarithmic. Furthermore, the scaling behavior $T_{N}-T_{F} \sim H^{2 / \phi}$ is observed with $\phi=1.74 \pm 0.02$, in good agreement with the random-field crossover exponent $12 \phi \approx$ 1.75. Hence, the instability of the long-range order is certainly connected with the randomfield behavior. Just as in the case of $d=3$, once the domains are introduced into the system below $T_{N}$ and the field is turned off, the domains remain for $T<T_{N}$ even though the ground state is long-range order.

The dynamics of domain formation at low temperatures have been studied very close to the percolation threshold in $R b_{2} C o_{0.60} M g_{0.40} F_{4}$ by Ikeda, et al. 100 using neutron and magnetization techniques. Currently, the behavior is being investigated 15 at higher concentration in $\mathrm{Rb}_{2} \mathrm{Co}_{0.85} \mathrm{Mg}_{0.15} \mathrm{~F}_{4}$.

\section{Griffiths-like Phase in Dilute Antiferromagnets}

Griffiths 101 showed that the magnetization in dilute magnets is nonanalytic in $H$ at $H=0$ below the transition temperature of the corresponding pure system. This is a consequence of 
the randomness of the local magnetic concentration. Evidence for dilution-induced Griffiths instabilities has been observed 0 by studying the deviations from the Curie-Weiss behavior of $\chi^{\prime}$ which appears at the pure Néel temperature and extends down to the transition temperature in $\mathrm{Fe}_{0.47} Z n_{0.53} F_{2}$ and $K_{2} C u_{0.8} Z n_{0.2} F_{2}$. A similar, but much stronger effect is observed in $F e_{0.47} Z n_{0.53} F_{2}$ once random fields are introduced. Binek and Kleemann 50.46 were able to describe the field-induced Griffiths-like peak in $\chi^{\prime}$, seen as the broad peak in Fig. 4, using a phenomenological Lorentzian density distribution of local critical temperatures between $T_{c}(0)=T_{N}$ and $T_{c}(H)$ with a corresponding power law $\chi^{\prime}$ behavior at each temperature. These phenomena have only recently been investigated 15 in $d=2$ systems.

\section{The $d=3$ RFIM at Large Magnetic Dilution and Large Fields}

New physics emerges once the percolation threshold $x \approx 0.24$ in $F e_{x} Z n_{1-x} F_{2}$ is approached. The system behaves much like a spin-glass 102.103, as was first discovered by Montenegro, et al.104-107. This behavior takes place even though the frustrating exchange interactions in $F e_{x} Z n_{1-x} F_{2}$ are very small 16 . Near the percolation threshold, even tiny frustrating interactions are predicted to become important 17 . For Ising systems, it is also expected that the dynamics even in zero field should be extremely slow 108. Both of these may contribute to the spin-glass-like behavior, although computer simulations seem to indicate that the small frustrating interactions are sufficient 14 . 2 . V. Very close to the percolation threshold, for $x=0.25$ and $x=0.27$, no Bragg peak, and hence no antiferromagnetic ordering, is observed in zero field with neutron scattering 106 . (Interestingly, this does not seem to have been observed in the related anisotropic system $109 C_{0.26} Z n_{0.74} F_{2}$ or in the weakly anisotropic system $110 M n_{x} Z n_{1-x} F_{2}$.) The antiferromagnetic correlation length $\xi$ remains small and constant for $T$ below approximately $10 \mathrm{~K}\left(T_{N}=78.4 \mathrm{~K}\right.$ for pure $\left.F e F_{2}\right)$. Mössbauer measurements indicate a competition between antiferromagnet and spin-glasslike order 111 . The temperature below which $\xi$ remains constant is just the endpoint of the de Almeida-Thouless line $T_{e q}(H)$. The $T_{c}(H)$ curvature is described well by a crossover exponent $\phi=3.4$, the same exponent measured in canonical spin-glasses 102 . For a higher concentration, $x=0.31$, a more complicated phase diagram is observed 104 . The lowfield behavior is the same as observed for higher concentrations, i.e. the low-field phase is antiferromagnetic and $\phi=1.42$. As the field increases, the curvature changes to $\phi=3.4$ and no antiferromagnetic order is observed below $T_{e q}(H)$. The large field induces the spin-glass-like behavior away from percolation. As we move to even higher concentrations, $x=0.5$, very high fields are needed to probe the region above the antiferromagnetic phase, as shown by Lima, et al.57 employing high-field magnetization measurements. Computer simulations 18 indicate that below $x \approx 0.6$, weak frustration affects the ordered state of the REIM in dilute antiferromagnets.

In the less anisotropic system $M n_{0.35} Z n_{0.65} F_{2}$, somewhat similar behavior to that in $F e_{0.31} Z n_{0.69} F_{2}$ is observed 112 in magnetization and ac susceptibility studies. There is some indication that the phase diagrams may differ in some respects and this is currently under investigation. A de Almeida-Thouless line with $\phi=3.4$ is observed for $M n_{0.35} Z n_{0.65} F_{2}$.

A spin-glass-like phase has also been observed above the mixed phase in $\mathrm{Fe}_{x} \mathrm{Mg}_{1-x} \mathrm{Cl}_{2}$ 
for relatively large magnetic concentrations 113 . Slow dynamics are observed for the metastable domain structure within the mixed antiferromagnetic-paramagnetic phase 19 . The memory of domain structure is preserved upon decreasing the field to zero and even upon field reversal. The memory effect is also observed after entering the spin-glass-like phase.

\section{First-order to Second-order Transition in $\mathrm{Fe}_{x} \mathrm{Mg}_{1-x} \mathrm{Cl}_{2}$}

Recently the metamagnetic transition in $\mathrm{Fe}_{x} \mathrm{Mg}_{1-x} \mathrm{Cl}_{2}$ for has been studied optically and

with computer simulations 114 . Rounding of the metamagnetic transition is interpreted as the driving of the transition from first-order to second-order by random fields and randomfield-induced domain structure. The domain structure is optically observed to be greatly altered by the dilution-induced random fields. This is in accord with predictions that quenched impurities 115 and random fields 116 can drive a phase transition from first-order to second-order. The concentration at which the metamagnetic transition becomes secondorder is estimated to be $x=0.6$. For sufficient dilution the first-order nature of the transition is lost when the avalanche of domain flipping no longer involves infinite length scales. Universal behavior is predicted for this nonequilibrium transition 117 .

\section{Other RFIM Systems}

Although a great deal of the experiments shedding light on the RFIM have been done on dilute antiferromagnets, other systems have been studied as well. Kleemann 118 has reviewed random-field domain states in ferroelectric and structural phase transitions. The critical behavior of the RFIM structural phase transition in $D y A s_{x} V_{1-x} O_{4}$ has been studied extensively 119 and compared to the dilute antiferromagnet. Neutron and light scattering experiments have been done on binary mixtures in silica gels 120 . Certainly more RFIM realizations will be studied in the future and will significantly add to our understanding as well as incorporate aspects of the dilute antiferromagnet results.

\section{Conclusions}

There is good reason to be optimistic about achieving a good characterization of $d=3$ RFIM critical behavior in the near future. Experiments are nearly at the point where serious comparisons between theory and experiment can be made. This is possible since the high concentration crystals show no evidence for the formation of domain structure or for hysteresis in the line shapes well below the transition, two aspects of the experiments at lower concentration that have been severe impediments. It would be interesting to investigate if the remarkable difference in the behavior at high and low magnetic concentration is a result of a concentration critical point below which the long-range order becomes unstable. One outstanding problem is the lack of a theoretically derived line shape to use in analyzing data; the mean-field arguments are clearly inadequate. When such a theory is developed, more reliable critical exponents and amplitude ratios will be derived from the scattering data. 
Two kinds of hysteresis can now be distinguished for $d=3$. At low magnetic concentrations, vacancies cause irreversibilities and domain formation which are most evident in scattering experiments. At all concentrations where transitions take place, there appears to be hysteresis, observable in all experiments, that may be attributable to random-field critical dynamics. For $d=2$, hysteresis occurs only at low temperatures, well below the rounded transition. The dynamics of domain formation in this region are still being studied.

Griffiths-like domain structure dominates the ac suseptibility in the $d=3$ random-field transition in $\mathrm{Fe}_{0.46} Z n_{0.54} F_{2}$. It remains a task to determine whether the small critical peak, recently discovered, yields power-law or activated dynamics.

Near the percolation threshold, it appears that the $d=3$ Ising system $F e_{x} Z n_{1-x} F_{2}$ behaves very much like a spin-glass despite having only small frustrating interactions. The behavior in the more isotropic $M n_{x} Z n_{1-x} F_{2}$ is being studied to elucidate the role of anisotropy in the spin-glass-like behavior. In related studies, intermediate concentration crystals of $F e_{x} Z n_{1-x} F_{2}$ are being studied in the high-field limit.

Recent experiments have addressed the random-field effects on first-order transitions in $\mathrm{Fe}_{x} \mathrm{Mg}_{1-x} \mathrm{Cl}_{2}$. The first-order transition appears to be driven to be second-order with sufficiently strong random fields, in agreement with theory.

A reasonable understanding of the random-field Ising model as realized in dilute antiferromagnets is emerging, though there is considerable work yet to be done. All aspects of the rich behavior of these dilute antiferromagnets are important to characterize partly for their intrinsically interesting properties and partly because other materials may show one or more of the characteristics. The antiferromagnets are the best studied and probably the most easily understood systems. In trying to understand the behavior in more complex systems, one will have to keep in mind the array of possible behaviors. Certainly, the random-field physics will be incorporated into the descriptions of many important materials in the future.

\section{Acknowledgements}

Some of the experiments on the $d=3 F e_{x} Z n_{1-x} F_{2}$ would not be possible without the extraordinary crystals produced by Vince Jaccarino, Allan King and Neil Nighman. I thank Vince Jaccarino for giving some of them to me. I would like to thank Zoran Slanič, Jaime Fernandez-Baca, Wolfgang Kleemann, Frederico Montenegro, Christian Binek and others for allowing me to review their data prior to publication and for interesting discussions. In addition to those above, I would like to acknowledge Bob Nicklow, Hideki Yoshizawa, Jiahua Wang, Seung-Jin Han, Keith Dow, Carlos Ramos, David Lederman, Mark Lui, Sergio Rezende, Peter Pollak, Ingrid Ferreira, and others with whom I enjoyed collaborating on some of the work reviewed here. I would like to thank Peter Young, Uli Nowak and others for interesting and helpful discussions. This work has been supported by Department of Energy Grant No. DE-FG03-87ER45324.

\section{References}


1. Y. Imry and S. K. Ma, Phys. Rev. Lett. 35, 1399 (1975).

2. M. Mézard and A. P. Young, Europhys. Lett. 18, 653 (1992); C. De Dominicis, H. Orland and T. Temesvari, J. Phys. I France 5, 987 (1995).

3. D. P. Belanger and A. P. Young, J. Mag. Mag. Mater. 100, 272 (1991).

4. D. P. Belanger in Recent Progress in Random Magnets, ed. D. H. Ryan (World Scientific, Singapore, 1992).

5. J. Bricmont and A. Kupiainen, Phys. Rev. Lett. 59, 1829 (1987); J. Z. Imbrie, Phys. Rev. Lett. 53, 1747 (1984).

6. J. L. Cardy, Phys. Rev. B 29, 505 (1984).

7. S. Fishman and A. Aharony, J. Phys. C 12, L729 (1979).

8. Z. Slanič, D. P. Belanger and J. A. Fernandez-Baca, submitted for publication (1996).

9. T. Nattermann and I. Vilfan, Phys. Rev. Lett. 61, 223 (1988).

10. J. R. L. de Almeida and D. Thouless, J. Phys. A 11, 983 (1978).

11. K. Binder, Phys. Rev. B 29, 5184 (1984); I. Morgenstern, K. Binder and R. M. Hornreich, Phys. Rev. B 23, 287 (1981).

12. I. B. Ferreira, A. R. King, V. Jaccarino, J. L. Cardy and H. J. Guggenheim, Phys. Rev. B 28, 5192 (1983).

13. H. Ikeda, J. Phys. C 16, L21 (1983); J. Phys. C 16, L1033 (1983).

14. U. Nowak, J. Esser and K. D. Usadel, to be published in Physica A, 1996.

15. C. Binek, C. Jakobs, W. Kleemann and D. P. Belanger, unpublished.

16. M. T. Hutchings, B. D. Rainford and H. J. Guggenheim, J. Phys. C 3, 307 (1970); C. B. Araújo Phys. Rev. B 22, 266 (1980).

17. B. W. Southern, A. P. Young and P. Pfeuty, J. Phys. C 12, 683 (1979).

18. E. P. Raposo, M. D. Coutinho-Filho and F. C. Montenegro, Europhys. Lett. 29, 507 (1995); J. Mag. Mag. Mater. 154, L155 (1996).

19. J. Mattsson, J. Kushauer, D. Bertrand, J. Ferré and W. Kleemann, J. Magn. Magn. Mater. 130, 216 (1994).

20. P.-z. Wong, S. von Molnar, T. T. M. Palstra, J. A. Mydosh, H. Yoshizawa, S. M. Shapiro and A. Ito, Phys. Rev. Lett. 55, 2043 (1985); D. Bertrand, A. R. Fert, M. C. Schmidt, F. Bemsamka and S. Legrand, J. Phys. C 15, L883 (1982); J. Phys. C 17, 1725 (1984).

21. P. W. Mitchell, R. A. Cowley, H. Yoshizawa, P. Böni, Y. J. Uemura and R. J. Birgeneau, Phys. Rev. B 34, 4719 (1986).

22. D. P. Belanger, A. R. King and V. Jaccarino, Phys. Rev. B 34, 452 (1986).

23. Y. Shapira, N. F. Oliveira, Jr. and S. Foner, Phys. Rev. B 30, 6639 (1984).

24. Y. Shapira, J. Appl. Phys. 53, 1931 (1982); Y. Shapira and N. F. Oliveira, Jr., Phys. Rev. B 27, 4336 (1983); F. C. Montenegro, J. C. O. de Jesus, F. L. A. Machado, E. Montarroyos and S. M. Rezende, J. Mag. Mag. Mater. 104-107, 277 (1992).

25. H. Ikeda and M. T. Hutchings, J. Phys. C 11, L529 (1978).

26. A. R. King, I. B. Ferreira, V. Jaccarino and D. P. Belanger, Phys. Rev. B 37, 219 (1988); D. P. Belanger, A. R. King, I. B. Ferreira and V. Jaccarino, Phys. Rev. B 37, $226(1988)$.

27. C. A. Ramos, A. R. King, and V. Jaccarino, Phys. Rev. B 37, 5483 (1988). 
28. M. F. Collins, Magnetic Critical Scattering, Oxford University Press, New York, 1989.

29. J. Villain, J. de Phys. 46, 1843 (1985).

30. D. S. Fisher, Phys. Rev. Lett. 56, 416 (1986).

31. A. Aharony and G. Ahlers, Phys. Rev. Lett. 44, 782 (1980).

32. D. P. Belanger and H. Yoshizawa, Phys. Rev. B 35, 4823 (1987) and references therein.

33. P.-z. Wong, S. von Molnar and P. Dimon, Sol. St. Comm. 48, 573 (1983).

34. P.-z. Wong, S. von Molnar and P. Dimon, J. Appl. Phys. 53, 7954 (1982).

35. D. P. Belanger, A. R. King, V. Jaccarino and J. L. Cardy, Phys. Rev. B 28, 2522 (1983).

36. I. B. Ferreira, A. R. King and V. Jaccarino, Phys. Rev. B 43, 10797 (1991); J. Appl. Phys. 69, 5246 (1991).

37. U. A. Leitão and W. Kleemann, Phys. Rev. B 29, 505 (1984); Phys. Rev. B 35, 8696 (1987).

38. A. Aharony, Europhys. Lett. 1, 617 (1986).

39. W. Kleemann, A. R. King and V. Jaccarino, Phys. Rev. B 34, 479 (1986).

40. U. A. Leitão, W. Kleemann and I. B. Ferreira, J. de Phys. C8, 1217 (1988).

41. R. J. Birgeneau, Q. Feng, Q. J. Harris, J. P. Hill, A. P. Ramirez and T. R. Thurston, Phys. Rev. Lett. 75, 1198 (1995); Phys. Rev. Lett. 77, 2342 (1996).

42. M. E. Fisher and J. S. Langer, Phys. Rev. Lett. 20, 665 (1968).

43. J. Ferré and G. A. Gehring, Rep. Prog. Phys. 47, 513 (1984).

44. K. E. Dow and D. P. Belanger, Phys. Rev. B 39, 4418 (1989).

45. It has recently been stated by P.-z. Wong, Phys. Rev. Lett. 77, 2340 (1996), that new data 41 using $F e_{0.5} Z n_{0.5} F_{2}$ show that the birefringence technique fails to show the true specific heat critical behavior. However, the technique works in the less anisotropic case of $M n_{x} Z n_{1-x} F_{2}$ for several concentrations 27 and should be even better 43 for $F e_{0.5} Z n_{0.5} F_{2}$. All concentrations of $F e_{x} Z n_{1-x} F_{2}$ that have been studied accurately enough to reveal critical behavior show consistent behavior as discussed in this review. The birefringence technique is in fact much more accurate than trying to subtract the large phonon contributions from the magnetic ones in the bulk crystal specific heat.

46. Ch. Binek, S. Kuttler and W. Kleemann, Phys. Rev. Lett. 75, 2412 (1995).

47. It has recently been claimed 41 that justification for seeing the antiferromagnetic order parameter in magnetization or birefringence measurements can be found in the work of Langer and Fisher 4 . However, that reference is the basis of seeing the critical behavior of the specific heat in resistivity measurements and the argument can be developed further 43 to show why birefringence exhibits the specific heat critical behavior. It does not address long-range antiferromagnetic order.

48. D. P. Belanger, W. Kleemann and F. C. Montenegro, Phys. Rev. Lett. 77, 2431 (1996).

49. P. Pollak, W. Kleemann and D. P. Belanger, Phys. Rev. B 38, 4773 (1988).

50. Ch. Binek and W. Kleemann, Phys. Rev. B 51, 12888 (1995).

51. A. B. Harris, J. Phys. C 7, 1671 (1974); G. Jug, Phys. Rev. B 27, 609 (1983);

K. E. Newman and E. K. Riedel, Phys. Rev. B 25, 264 (1982). 
52. A. R. King, V. Jaccarino, D. P. Belanger and S. M. Rezende, Phys. Rev. B 32, 503 (1985).

53. M. Mézard and R. Monasson, Phys. Rev. B 50, 7199 (1994); J. R. L. de Almeida and R. Bruinsma, Phys. Rev. B 35, 7267 (1987).

54. H. Yoshizawa and D. P. Belanger, Phys. Rev. B 30, 5220 (1984); C. M. Soukoulis, G. Grest, C. Ro and K. Levin, J. Appl. Phys. 57, 3300 (1985); G. Grest, C. M. Soukoulis and K. Levin, Phys. Rev. B 33, 7659 (1986).

55. H. Ikeda, J. Phys. C 19, L811 (1986); H. Ikeda and K. Kikuta, J. Phys. C 17, 1221 (1984).

56. P.-z. Wong and J. W. Cable, Phys. Rev. B 28, 5361 (1983).

57. K. A. Lima, A. Lacerda and F. C. Montenegro, to be published.

58. M. Kaufman and M. Kardar, Phys. Rev. B 31, 2913 (1985).

59. H. Rieger and A. P. Young, J. Phys. A 26, 5279 (1993).

60. H. Rieger, Phys. Rev. B 52, 6659 (1995).

61. D. P. Belanger, A. R. King and V. Jaccarino, Phys. Rev. Lett. 48, 1050 (1982); J. Appl. Phys. 53, 2702 (1982).

62. H. Yoshizawa, R. A. Cowley, G. Shirane, R. J. Birgeneau, H. J. Guggenheim, and H. Ikeda, Phys. Rev. Lett. 48, 438 (1982).

63. D. P. Belanger, A. R. King and V. Jaccarino, Sol. St. Comm. 54, 79 (1985).

64. D. P. Belanger, Phase Transitions 11, 53 (1988).

65. S. W. Lovesey, J. Phys. C 17, L213 (1984); P. M. Richards, Phys. Rev. B 30, , (2955)1984; P. Debye, H. R. Anderson, Jr. and H. Brumberger, J. Appl. Phys. 28, 679 (1957).

66. R. A. Pelcovits and A. Aharony, Phys. Rev. B 31, 350 (1985).

67. M. E. Fisher and R. J. Burford, Phys. Rev. 156, 583 (1967); H. B. Tarko and M. E. Fisher, Phys. Rev. B 11, 1217 (1975); C. A. Tracy and B. McCoy, Phys. Rev. B 12, 368 (1975); R. A. Cowley, M. Hagen and D. P. Belanger, J. Phys. C 17, 3763 (1984); M. Hagen, R. A. Cowley, R. M. Nicklow and H. Ikeda, Phys. Rev. B 36, 401 (1087).

68. D. P. Belanger, A. R. King, V. Jaccarino, and R. M. Nicklow, Phys. Rev. Lett. 59, 930 (1987).

69. D. P. Belanger, A. R. King and V. Jaccarino, Phys. Rev. B 31, 4538 (1985).

70. R. A. Cowley, H. Yoshizawa, G. Shirane, M. Hagen and R. J. Birgeneau, Phys. Rev. B 30, 6650 (1984).

71. D. P. Belanger, J. Wang, Z. Slanič, S-J. Han, R. M. Nicklow, M. Lui, C. A. Ramos and D. Lederman, Phys. Rev. B 54, 3420 (1996).

72. M. Lui, A. R. King, V. Jaccarino and G. L. Snider, Phys. Rev. B 40, 4898 (1989); M. Lui, thesis (unpublished), UCSB (1989).

73. U. Nowak and K. D. Usadel, Phys. Rev. B 44, 7426 (1991); Phys. Rev. B 46, 8329 (1992); Physica A 191, 203 (1992); Phys. Rev. B 43, 851 (1991); S-J. Han and D. P. Belanger, Phys. Rev. B 46, 2926 (1992); S-J. Han, D. P. Belanger, W. Kleemann and U. Nowak Phys. Rev. B 45, 9728 (1992).

74. U. A. Leitão, W. Kleemann and I. B. Ferreira, Phys. Rev. B 38, 4765 (1988). 
75. J. P. Hill, Q. Feng, R. J. Birgeneau, and T. R. Thurston, Phys. Rev. Lett. 70, 3655 (1993); Z. Phys. B 92, 285 (1993).

76. C. Magon, J. Sartorelli, A. R. King, V. Jaccarino, M. Itoh, H. Yasuoka and P. Heller, J. Mag. Mag. Mater. 54-57, 49 (1986).

77. R. J. Birgeneau, R. A. Cowley, G. Shirane and H. Yoshizawa, Phys. Rev. Lett. 54, 2147 (1985).

78. A. P. Young and M. Nauenberg, Phys. Rev. Lett. 54, 2429 (1985); A. Falicov, A. N. Berker and S. R. McKay, Phys. Rev. B 51, 8266 (1995); A. T. Ogielski, Phys. Rev. Lett. 57, 1251 (1986); I. Dayan, M. Schwartz and A. P. Young, J. Phys. A 26, 3093 (1993); M. S. Cao and J. Machta, Phys. Rev. B 48, 3177 (1993); Y. Shapir, Phys. Rev. B 35, 62 (1987); Y. Shapir, Phys. Rev. Lett. 54, 154 (1985).

79. C. A. Ramos, A. R. King, V. Jaccarino and S. M. Rezende, J. de Phys Coll. C8 49, 1241 (1988).

80. D. Lancaster, E. Marinari and G. Parisi, J. Phys. A 28, 3959 (1995).

81. R. A. Cowley, R. J. Birgeneau and G. Shirane, Physica 140A, 285 (1986).

82. M. Gofman, J. Adler, A. Aharony, A. B. Harris and M. Schwartz, Phys. Rev. Lett. 71, 1569 (1993); Phys. Rev. B 53, 6362 (1996).

83. D. P. Belanger, B. Farago, V. Jaccarino, A. R. King, C. Lartigue and F. Mezei, J. de Phys. C 8, 1229 (1988).

84. H. Rohrer, J. Appl. Phys. 52, 1708 (1981).

85. A. R. King, J. A. Mydosh and V. Jaccarino, Phys. Rev. Lett. 56, 2525 (1986).

86. A. E. Nash, A. R. King and V. Jaccarino, Phys. Rev. B 43, 1272 (1991).

87. Y. S. Parmer and J. K. Bhattacharjee, Phys. Rev. B 49, 6350 (1994).

88. V. Dotsenko, J. Phys. A 27, 3397 (1994).

89. W. Kleemann, B. Igel and U. A. Leitão in New Trends in Magnetism, ed. M. D. Coutinho-Filho and S. M. Rezende (World Scientific, Singapore, 1990).

90. M. Hagen, R. A. Cowley, R. M. Nicklow and H. Ikeda, Phys. Rev. B 36, 401 (1987).

91. P. M. C. de Oliveira, S. M. M. de Oliveira and S. L. A. de Queiro, Physica A 175, 345 (1991).

92. C. Dekker,B. J. Dikken and A. F. M. Arts, Sol. St. Comm. 54, 887 (1985).

93. R. J. Birgeneau, H .Yoshizawa, R. A. Cowley, G. Shirane and H. Ikeda, Phys. Rev. B 28, 1438 (1983).

94. D. P. Belanger, A. R. King and V. Jaccarino, unpublished.

95. M. Lederman, J. V. Selinger, R. Bruinsma, R. Orbach and J. Hammann, Phys. Rev. B 48, 3810 (1993); M. Lederman, J. V. Selinger, R. Bruinsma, J. Hammann and R. Orbach, Phys. Rev. Lett. 68, 2086 (1992).

96. J. Villain, Phys. Rev. Lett. 52, 1543 (1984).

97. Q. Feng, R. J. Birgeneau and J. P. Hill, Phys. Rev. B 51, 15188 (1995).

98. E. Oğuz, J. Phys. A 27, 2985 (1994).

99. D. P. Belanger, A. R. King and V. Jaccarino, Phys. Rev. Lett. 54, 577 (1985).

100. H. Ikeda, Y. Endo and S. Itoh, Phys. Rev. Lett. 64, 1266 (1990).

101. R. B. Griffiths, Phys. Rev. Lett. 23, 17 (1969).

102. K. Binder and A.P. Young, Rev. Mod. Phys. 58, 801 (1986). 
103. H. Yoshizawa, S. Mitsuda, H. Aruga and A. Ito, Phys. Rev. Lett. 59, 2364 (1987).

104. F. C. Montenegro, U. A. Leitão, M. D. Coutinho-Filho and S. M. Rezende, J. Appl. Phys. 67, 5243 (1990); F. C. Montenegro, A. R. King, V. Jaccarino, S-J. Han and D. P. Belanger, Phys. Rev. B 44, 2155 (1991); D. P. Belanger, W. E. Murray, Jr., F. C. Montenegro, A. R. King, V. Jaccarino and R. W. Erwin, Phys. Rev. B 44, 2161 (1991).

105. F. C. Montenegro, M. D. Coutinho-Filho and S. M. Rezende, J. Appl. Phys. 63, 3755 (1989); F. C. Montenegro, M. D. Coutinho-Filho and S. M. Rezende, Europhys. Lett. 8, 382 (1989); S. M. Rezende, F. C. Montenegro, M. D. Coutinho-Filho, C. C. Becerra and A. Paduan-Filho, J. Phys. (Paris) C8 49, 1267 (1989). S. M. Rezende, F. C. Montenegro, U. A. Leitão and M. D. Coutinho-Filho in New Trends in Magnetism, ed. M. D. Coutinho-Filho and S. M. Rezende (World Scientific, Singapore, 1990).

106. D. P. Belanger and H. Yoshizawa, Phys. Rev. B 47, 5051 (1993).

107. D. P. Belanger, thesis, UCSB, 1981.

108. C. L. Henley, Phys. Rev. Lett. 54, 2030 (1985).

109. M. Hagen, R. A. Cowley, S. K. Satija, H. Yoshizawa, G. Shirane, R. J. Birgneneau and H. J. Guggenheim, Phys. Rev. B 28, 2602 (1983).

110. R. A. Cowley, G. Shirane, R. J. Birgeneau, E. C. Svensson and H. J. Guggenheim, Phys. Rev. B 22, 4412 (1980).

111. J. H. de Araujo, J. B. M. da Cunha, A. Vasquez, L. Amaral, J. T. Moro, F. C. Montenegro, S. M. Rezende and M. D. Coutinho-Filho, Rev. Brasil. de Física 21, 115 (1991); J. B. M. da Cunha, J. H. de Araujo, L. Amaral, A. Vasquez, J. T. Moro, F. C. Montenegro, S. M. Rezende and M. D. Coutinho-Filho, Hyper. Int. 54, 489 (1990).

112. F. C. Montenegro, J. C. O. de Jesus and A. Rosales-Rivera, J. Appl. Phys. 75, 5520 (1994); F. C. Montenegro, A. Rosales-Rivera, J. C. O. de Jesus, E. Montarroyos and F. L. A. Machado, Phys. Rev. B 51, 5849 (1995); F. C. Montenegro, Z. Slanič, D. P. Belanger and J. A. Fernandez-Baca, unpublished.

113. D. Bertrand, J. Ferré, P. Meyer, J. Pommier and W. Kleemann, J. Mag. Mag. Mater. 104-107, 389 (1992); J. Mattsson, J. Kushauer, D. Bertrand, J. Ferré, J. Pommier, P. Meyer and W. Kleemann, J. Mag. Mag. Mater. 152, 129 (1996).

114. J. Kushauer, R. van Bentum, W. Kleemann and D. Bertrand, Phys. Rev. B 53, 11647 (1996); J. Kushauer and W. Kleemann, J. Mag. Mag. Mater. 140-144, 1551 (1995).

115. Y. Imry and M. Wortis, Phys. Rev. B 19, 3580 (1979).

116. J. P. Sethna, K. Dahmen, S. Kartha, J. A. Krumhansl, B. W. Roberts and J. D. Shore, Phys. Rev. Lett. 70, 3347 (1993); K. Dahmen, S. Kartha, J. A. Krumhansl, B. W. Roberts, J. P. Sethna and J. D. Shore, J. Phys. A 75, 5946 (1994).

117. K. Dahmen and J. P. Sethna, Phys. Rev. B 53, 14872 (1996).

118. W. Kleemann, Int. J. Mod. Phys. B 7, 2469 (1993).

119. K. A. Reza and D. R. Taylor, Phys. Rev. B 46, 11425 (1992); J. T. Graham, J. H. Page and D. R. Taylor, Phys. Rev. B 44, 4127 (1991); H. M. Elmehdi, J. H. Page and J. T. Graham, J. Magn. Magn. Mater. 104-107, 193 (1992); J. T. Graham, 
D. R. Taylor, D. R. Noakes, and W. J. L. Buyers, Phys. Rev. B 43, 3778 (1991); D. R. Taylor, K. A. Reza and J. T. Graham, Phys. Rev. B 52, 7108 (1995); D. R. Taylor and K. A. Reza, J. Phys. Cond. Matt. 6, 10171 (1994); Z. Slanič, D. P. Belanger, J. Wang and D. R. Taylor, Phys. Rev. B 53, 97 (1996).

120. B. J. Frisken, D. S. Cannell, M. Y. Lin and S. K. Sinha, Phys. Rev. E 51, 5866 (1995); B. J. Ferri and D. S. Cannell, Phys. Rev. E 51, 5922 (1995). 